\title{
Thyroid dysfunction in rheumatoid arthritis: a controlled prospective survey
}

\author{
Jeffrey B Shiroky, Martin Cohen, Marie-Louise Ballachey, Carolyn Neville
}

\begin{abstract}
Objectives-To determine whether thyroid dysfunction is found with increased frequency in patients with rheumatoid arthritis (RA).

Methods-A controlled prospective survey was conducted on a cohort of patients with RA derived from a hospital clinic and a private surburban rheumatology practice. A control group with similar demographic features was generated from the same sources and included subjects with either osteoarthritis or fibromyalgia. Consecutive patients were evaluated over a six month period. The evaluation included a complete history and physical examination, and determination of serum thyroxine, free thyroxine, triiodothyronine, thyroid stimulating hormone (IRMA), antinuclear antibodies, and rheumatoid factor.

Results-Of the 91 women with RA evaluated, $29(30 \%)$ had evidence of thyroid dysfunction compared with 10 $(11 \%)$ of 93 controls. The excess thyroid dysfunction is due to either hypothyroidism or Hashimoto's thyroiditis and was independent of age, increasing duration of disease, rheumatoid factor, and antinuclear antibodies.

Conclusions-Thyroid dysfunction is seen at least three times more often in women with $R A$ than in women with similar demographic features with noninflammatory rheumatic diseases such as osteoarthritis and fibromyalgia.
\end{abstract}

(Ann Rheum Dis 1993; 52: 454-456)

Division of

Rheumatology,

Montreal General

Hospital,

Montreal, Quebec,

Canada

J B Shiroky

$M$ Cohen

M-L Ballachey

C Neville

Correspondence to: Dr J B Shiroky,

Division of Rheumatology,

Montreal General Hospital,

1650 Cedar Avenue,

Montreal, Quebec,

Canada H3G 1A4.

Accepted for publication

1 February 1993 orders such as rheumatoid arthritis (RA) and autoimmune thyroid disease. In several surveys conducted since the early 1960 s a relation was suggested to exist between Hashimoto's thyroiditis and RA. ${ }^{1-4}$ The surveys were criticised as being uncontrolled, using too small a sample size, or possibly being affected by a Berksonian bias-that is, patients seen in a referral centre (e.g. a hospital based clinic) are more likely to have more than one disease at the time of referral than a patient followed in the community.
One controlled population based study did not identify an association between thyroiditis and RA, but can be considered flawed as it is a retrospective chart review with an unclear case definition. ${ }^{5}$ Two further studies, one retrospective at necropsy and the other a small clinical study, did not identify a strong association. ${ }^{6} 7$

In spite of these results, the relation has repeatedly surfaced, with workers at several centres noting that thyroid disease is common in family members of patients with RA, with a genetic association apparently independent of HLA-DR4 ${ }^{8-10}$ In view of this, we began a prospective controlled survey of our patients with RA for the presence of thyroid dysfunction. Our survey suggests that thyroid dysfunction is found more often in patients with RA than in subjects seen in the same setting with non-inflammatory rheumatic diseases.

\section{Subjects and methods}

PATIENTS

To avoid a Berksonian bias, consecutive patients seen over a six month period were recruited from two sources: the rheumatology outpatient clinic of a university hospital (Montreal General Hospital) and a suburban community based rheumatology practice 10 miles away. All patients with RA fulfilled the criteria of the American College of Rheumatology. ${ }^{11}$ A control group was selected from patients seen in the same settings with either osteoarthritis or primary fibromyalgia; two non-inflammatory rheumatic diseases. Having prior knowledge of the demographic features of the patients, a control group with a ratio of 1:2 fibromyalgia to osteoarthritis would give the same age distribution as our patients with RA. All the patients lived within 75 miles of Montreal. This patient group was approximately $90 \%$ white, with a few black, oriental, Amerindian, and east Indian patients.
EVALUATION of thyroid disease, use of thyroid drugs or supplementation, and blood samples were obtained for the measurement of thyroxine, free thyroxine, triiodothyronine, and thyroid stimulating hormone (IRMA). Samples of blood were also obtained for the detection of rheumatoid factor (nephelometry) and
Each patient was evaluated for a history 
antinuclear antibodies (immunofluorescence analysis on Hep-2 cells). An abnormal level of thyroid stimulating hormone was defined as $>6 \mathrm{mU} / 1$ or $<0.5 \mathrm{mU} / 1$.

When a history of thyroid disease was reported, confirmation was made by either chart review or by contacting the appropriate doctor for the actual diagnosis and how it was made. Unconfirmed cases were excluded. Only cases of Hashimoto's thyroiditis proved by biopsy were classified as such. Other cases of Hashimoto's thyroiditis unconfirmed by biopsy are probably included in the subset classified as hypothyroid.

\section{ANALYSIS}

Patient groups were compared by Pearson's $\chi^{2}$ test. An odds ratio was calculated for the frequency of thyroid dysfunction in patients with RA compared with controls and a 95\% confidence interval was calculated.

\section{Results}

One hundred and nineteen patients with RA were evaluated. Ninety one were women and 28 were men. One hundred and eight controls were evaluated. Ninety three were women, with 34 and 59 having fibromyalgia and osteoarthritis respectively. All patients with RA were receiving treatment with at least one disease modifying drug (hydroxychloroquine, gold, methotrexate, sulphasalazine, azathioprine or D-penicillamine).

Evidence for thyroid disease was obtained in $27 / 91$ (30\%) women with RA and 2/28 (7\%) men. There was one unconfirmed case of thyroid disease in a woman with RA. In the control group, 10/93 (11\%) women had evidence of thyroid disease. No men had thyroid disease and there were two unconfirmed cases. The two unconfirmed cases had osteoarthritis, normal laboratory results, and reported short courses of thyroid supplementation in the past. Six $(10 \%)$ patients with osteoarthritis and four $(12 \%)$ patients with fibromyalgia had thyroid disease.

When contrasting women with RA with the control women for the occurrence of thyroid disease, the results were statistically significant $(p>0.001)$ with an odds ratio of $3.5(95 \%$ confidence interval 1.62 to $7 \cdot 53$ ). The excess thyroid disease between groups appeared to be entirely due to either hypothyroidism or confirmed Hashimoto's thyroiditis (table 1). Of further interest, 10/27 (37\%) patients with $\mathrm{RA}$ with thyroid disease were diagnosed at the time of the survey by detecting increased levels of thyroid stimulating hormone and normal

Table 1 Types of thyroid disease identified in this study

\begin{tabular}{lll}
\hline & Patients with $R A^{*}$ & Controls \\
\hline Hypothyroidism $\dagger$ & 18 & 6 \\
Confirmed Hashimoto's thyroiditis & 6 & 1 \\
Graves' disease & 1 & 1 \\
Other diseases (multinodular goitre; familial) & 2 & 2 \\
\hline
\end{tabular}

$\star R A=$ rheumatoid arthritis

†May also include unconfirmed Hashimoto's thyroiditis.
Table 2 Comparison of the mean (range) age of patients with rheumatoid arthritis $(R A)$ and control subjects

\begin{tabular}{lll}
\hline & $\begin{array}{l}\text { Women } \\
\text { with } R A\end{array}$ & $\begin{array}{l}\text { Control } \\
\text { subjects }\end{array}$ \\
\hline All subjects & $58 \cdot 7(21-84)$ & $58 \cdot 8(29-86)$ \\
Subjects with thyroid disease & $58 \cdot 1(21-84)$ & $58 \cdot 3(37-81)$ \\
\hline
\end{tabular}

$\mathrm{p}=\mathrm{NS}$ in all instances.

thyroxine (confirmed by a repeated test) compared with only $1 / 10(10 \%)$ controls Control women and patients with RA were similar in age (table 2).

Women with RA were further contrasted for demographic differences. Those with thyroid disease did not differ in age, rheumatoid factor status, nor antinuclear antibody status. Those with thyroid disease tended to have a shorter duration of arthritis than those without thyroid disease (table 3). The presence of thyroid disease did not differ between the clinic and private practice (28 $v 32 \%$ ).

\section{Discussion}

Our results support an association between thyroid disease and RA. Thyroid disease was found in our female patients with $R A$ almost three times more often than in demographically similar control women. Hypothyroidism or Hashimoto's thyroiditis accounted for the excess thyroid disease in our female patients with RA. We did not have enough male patients with thyroid disease to draw conclusions about this subset.

To control for referral bias the controls were obtained from the same clinical sources. Patients with non-inflammatory rheumatic disease were used as their diseases were not thought to be associated with autoimmune thyroid disease. The frequency of thyroid dysfunction in our patients with osteoarthritis or fibromyalgia was not very different from each other. Controls with non-inflammatory disease were also used because of the possibility of identifying patients with thyroid dysfunction secondary to a systemic illness (sick euthyroid) such as RA. Such thyroid dysfunction is actually identified by a low level of thyroxine and normal thyroid stimulating hormone. None of our patients with RA or controls showed this abnormality on testing.

Of interest, $37 \%$ of our patients with RA and thyroid dysfunction were identified by an increased level of thyroid stimulating hormone at the time of the survey and had no previous history of thyroid disease. This probably reflects a previous underevaluation of thyroid disease in our patient population. The clinical significance of the increased results for thyroid stimulating hormone is unknown. Hypothyroidism, however, is associated with fatigue, anaemia, arthralgias/arthritis, and myalgias, all symptoms which would normally be attributed to the inflammatory state of a patient with RA. Furthermore, some workers have reported evidence for 'end-organ' metabolic abnormalities progressing on a continuum from an abnormal thyroid stimulating hormone test to overt clinical hypothyroidism. ${ }^{12}$ Thus the 
Table 3 Demographic features of women with rheumatoid arthritis

\begin{tabular}{llll}
\hline & Thyroid disease & No thyroid disease & $p$ Value \\
\hline Age (years) & $58 \cdot 1$ & $59 \cdot 0$ & NS \\
Mean (range) disease duration (years) & $8 \cdot 6(1-40)$ & $13 \cdot 5(1-44)$ & $0 \cdot 03$ \\
Rheumatoid factor (\%) & $44 \cdot 4$ & $50 \cdot 0$ & NS \\
ANA (\%)* & $44 \cdot 4$ & 43.8 & NS \\
\hline
\end{tabular}

*ANA=antinuclear antibodies

increases seen in our patients with $R A$, in the absence of abnormal thyroxine levels, may be clinically important. Other workers have suggested that thyroid dysfunction might exacerbate rheumatoid disease ${ }^{13}$ and a destructive arthropathy, mainly of the proximal interphalangeal joints, has been described in patients with primary hypothyroidism. ${ }^{14}$

Finally, it is assumed that an association between thyroid disease and RA is the result of a shared genetic predisposition to autoimmunity. This may not be true. Workers in Finland have observed an overrepresentation of RA in a well studied group of patients with non-autoimmune endemic goitre. ${ }^{15}$ As we assigned a diagnosis of Hashimoto's thyroiditis only to patients with biopsy proved disease, Hashimoto's thyroiditis could account for many of our other hypothyroid patients.

In summary, this study confirms that thyroid dysfunction occurs in patients with RA at a much higher frequency than comparable controls. The presence of thyroid dysfunction is unrelated to increasing age, duration of RA, rheumatoid factor status, or antinuclear antibody status. The excess thyroid dysfunction is either due to hypothyroidism or Hashimoto's thyroiditis.

We acknowledge the assistance of Ms C Kriticos in the preparation of this manuscript. This work was partially supported by a grant from the Arthritis Society of Canada.
Dr Shiroky is the recipient of a research scholarship from the Arthritis Society of Canada.

1 Buchanan W W, Crooks J, Alexander W D, Koutras D A Wayne E J, Gray K G. Association of Hashimoto' thyroiditis and rheumatoid arthritis. Lancet 1961; is $245-8$.

2 Buchanan W W. The relationship of Hashimoto's thyroiditis to rheumatoid arthritis. Geriatrics 1965; 20: 941-8.

3 Gibberd F B. A survey of four hundred and six cases of rheumatoid arthritis. Acta Rheumatol Scand 1965; 11: $62-70$.

4 Raymond J P, Kahn M F, Bourgeois P, et al. Etude prospective controllée des paramètres thyroïdiens prospective controllee des parametres thyroïdiens Rev Rhum Mal Osteoartic 1985; 52: 317-21.

5 Linos A, Worthington JW, Palumbo PJ, O'Fallon $M$ Kurland L T. Occurrence of Hashimoto's thyroiditis and diabetes mellitus in patients with rheumatoid arthritis. fChronic Dis 1980; 33: 73-7.

6 Masi A T, Hartman W H, Hahn B H, Abbey $\mathrm{H}$ Shulman L E. Hashimoto's disease. A clinicopathologica study with matched controls. Lancet 1965; i: 123-6.

7 Mulhern L M, Masi A T, Shulman L E. Hashimoto's disease. A search for associated disorders in 170 clinically detected cases. Lancet 1966; ii: 508-12.

8 Thomas D J B, Young A, Gorsuch A N, Bottazzo G F, Cudworth A G. Evidence for an association between rheumatoid arthritis and autoimmune endocrine disease. Ann Rheum Dis 1983; 42: 297-300.

9 Grennan D M, Dyer P A, Clague R, Dodds W, Smeaton I, Harris R. Family studies in RA-the Smeaton I, Harris R. Family studies in RA-the
importance of HLA-DR4 and of genes for autoimmune thyroid disease. $\mathcal{F}$ Rheumatol 1983 ; 10: 584-9.

10 Silman A J, Ollier W E R, Bubel M A. Autoimmune thyroid disease and thyroid autoantibodies in rheumatoid arthritis patients and their families. Br $\mathcal{F}$ Rheumatol 1989; 28 $18-21$

11 Arnett F C, Edworthy S M, Bloch D A, et al. The American Rheumatism Association 1987. Revised criteria for the classification of rheumatoid arthritis. Arthritis Rheum 1988; 31: 315-24.

12 Staub J-J, Althaus B U, Engler H, Ryff A S, et al. Spectrum of subclinical and overt hypothyroidism: effect of of subclinical and overt hypothyroidism: effect thyrotropin, prolactin and thyroid reserve and metabolic impact on peripheral target tissues. Am f Med 1992; 92

13 Delamere J P, Scott D L, Felix-Davies D D. Thyroid dysfunction and rheumatic diseases. $\mathcal{f} R$ Soc Med 1982; 75: $102-6$

14 Gerster J C, Valceschini P. Destructive arthropathy of fingers in primary hypothyroidism without chondrocalcinosis. Report of 3 cases. $\mathcal{F}$ Rheumatol 1992; 19 637-41.

15 Wahlberg P, Nyman D, Carlsson S A. 25 year follow-up of the Aland thyroid study of 1956 . Thyroid status and incidence of rheumatoid arthritis. Acta Endocrinol (Copenh) 1983; 251 (suppl): 47-52. 